\title{
Participación política de mujeres indígenas en tiempos de la Revolución Ciudadana*
}

\author{
LIZETH PÉREZ CÁRDENAS**
}

\begin{abstract}
Political Participation of Indigenous Women in Times of the Citizens' Revolution. The article analyzes the different ways in which indigenous women in Ecuador occupy decision-making positions. A journey of indigenous mobilization and new configurations of political participation of the indigenous women is presented during the period of the so-called Citizen's Revolution, led by Rafael Correa (2007-2017), in a scenario characterized by neo-extractivist policies that jeopardize territories and identities, contradicting the progress and the recognition of the rights achieved in the Constitution of 2008.
\end{abstract} Key words: Ecuador, ethnic identities, citizenship, indigenous movement and gender

\begin{abstract}
Resumen
El artículo analiza las formas en que las mujeres indígenas en Ecuador se insertan en espacios de toma de decisiones. Se presenta un recorrido sobre la movilización indígena y las nuevas configuraciones de la participación política de las mujeres indígenas en el periodo de la denominada Revolución Ciudadana encabezada por Rafael Correa (2007-2017), en un escenario caracterizado por políticas neoextractivistas que atentan contra los territorios y las identidades, en contradicción con los avances y el reconocimiento de derechos alcanzados en la Constitución de 2008.
\end{abstract}

Palabras clave: Ecuador, identidades étnicas, ciudadanía, movimiento indígena y género

\section{Introducción}

$\mathrm{L}$ a participación política de las mujeres en general y de las indígenas en particular se ha constituido como un elemento central en la consolidación de las llamadas democracias contemporáneas (Canabal, 2003; Palacios, 2005; Zegada, 2012). En las tres últimas décadas, el fortalecimiento y promoción de liderazgos de mujeres indígenas ha sido una de las materias pendientes de gobiernos, organizaciones y el movimiento indígena, que han incorporado a sus agendas estos procesos, reconociendo el papel estratégico de las mujeres en la ocupación de cargos en diferentes ámbitos. En Ecuador, las indígenas han sido parte de la movilización y han estado presentes en los diversos actos de contención que sus pueblos han desplegado frente al Estado; pese a que la intervención de las indígenas no ha sido mínima, sí ha sido invisibilizada y reducida, lo que las ha obligado a conformar nuevas organizaciones y a posicionar sus demandas dentro y fuera de sus comunidades, rescatando la voz de sus pueblos y nacionalidades.

Las condiciones particulares que enfrentan las indígenas las colocan tanto como "el último eslabón en la cadena de subordinaciones" (De la Cadena, 1991: 10), como los personajes donde la capacidad transformadora de la cultura se expresa con mayor limitación, al ser quienes generalmente permanecen en el ámbito rural

\footnotetext{
* Artículo recibido el 05/04/17 y aceptado el 21/08/17.

** Doctorado en Ciencias Antropológicas, Universidad Autónoma Metropolitana, Unidad Iztapalapa. Av. San Rafael Atlixco núm. 186, col. Vicentina, del. Iztapalapa, 09340, Ciudad de México. ORCiD: http://orcid.org/0000-0002-1385-2398
} 
y encuentran mayores dificultades para acceder a oportunidades. Al mismo tiempo que sufren una serie de exclusiones debido a su pertenencia étnica y de género, se enfrentan a concepciones androcéntricas ${ }^{1}$ y occidentales ${ }^{2}$ de la política, presentes en los espacios formales y en la vida comunitaria, hecho que obstaculiza su participación. Sin embargo, son sujetos políticos que ejercen el poder de distintas maneras, las cuales van más allá de las formas institucionales imaginadas por el Estado. Una de las conquistas sustantivas de las mujeres fue el derecho al voto, lo que les brindó la posibilidad de ser votadas y ocupar cargos públicos, así como su incorporación y participación en organizaciones y movimientos sociales, a través de los cuales posicionan sus exigencias como integrantes del movimiento étnico y como parte del movimiento de mujeres.

Tras un conjunto de avances significativos, un innegable reconocimiento, las transformaciones producto de la Constituyente de $2008^{3}$ y la denominada Revolución Ciudadana, los pueblos indígenas, y en especial sus mujeres, se ven afectados por políticas públicas que atentan contra sus territorios e identidades, lo cual impacta de forma directa en el ejercicio de sus derechos. Así, el objetivo de este artículo es identificar las peculiaridades de la participación de las mujeres indígenas, quienes se insertan en procesos con múltiples contradicciones cuando ocupan cargos en las instituciones del Estado y al interior de sus organizaciones. Por ello, es necesario visibilizar los elementos que fortalecen el ejercicio de derechos, permitiendo que las indígenas intervengan en los ámbitos locales y nacionales; sin dejar de lado la exclusión que enfrentan cuando desempeñan cargos dirigenciales.

Aquí presento ${ }^{4}$ algunos resultados de mi investigación de maestría sobre ciudadanía de mujeres kichwas en espacios de participación local en el cantón de Cotacachi, provincia de Imbabura, durante el periodo 2013-2014. En este proyecto utilicé herramientas de la metodología cualitativa, donde la observación participante fue fundamental para comprender los testi- monios y las 12 entrevistas semiestructuradas que realicé a miembros del movimiento indígena y lideresas comunitarias. Asimismo, revisé material bibliográfico, datos en medios de comunicación, y mantuve conversaciones con algunas lideresas indígenas en el contexto del proceso electoral de 2017, en el cual se elegiría presidente de la República y se renovaría la totalidad de los escaños en la Asamblea Nacional. ${ }^{5}$

El artículo está organizado en cuatro apartados en los cuales ubico cómo se ha conformado la participación de las mujeres indígenas. En el primero contextualizo y presento los antecedentes de la movilización indígena, en el segundo preciso las formas en que se configuró la participación política, en el tercero muestro ciertas características que han matizado el periodo de la Revolución Ciudadana, y en el cuarto busco indagar en algunas de las problemáticas y dificultades que las indígenas han encontrado en sus experiencias de ejercicio político.

Al igual que en otros países de América Latina, desde la Colonia y la formación del Estado nacional, los pueblos indígenas en Ecuador han sido víctimas de despojos, masacres, etnocidio, tortura y una serie de políticas encaminadas a la asimilación o al exterminio (Segato, 2011); por ello, según señalan De la Cadena y Starn (2009), hace un siglo era impensable que los indígenas fueran una fuerza activa; incluso podríamos decir que hace más de tres décadas era difícil imaginar el giro multicultural, el cual consistió en el reconocimiento de derechos a los pueblos indígenas. Este cambio discursivo y político no podría entenderse sin las amplias movilizaciones y acciones políticas de pueblos y comunidades, de tal modo que, en el proceso de lucha, los pueblos indígenas se fueron constituyendo como actores políticos, con una agenda propia, desde la que cuestionan marcos normativos y políticas económicas y públicas dirigidas a ellos.

Como parte del movimiento indígena, las mujeres han sido muy activas en los procesos de lucha de sus pueblos y han construido y pugnado de forma paralela por su propia agenda en cuanto mujeres ${ }^{6}$ (Hernández, 2008; Pequeño, 2009; Segato, 2011); esto las

1 Los hombres suelen ser los portavoces de las demandas del conjunto de la sociedad, por lo cual Pateman (2009) señala que las mujeres son vistas como objetos y no como sujetos de derechos.

2 Se entiende a la política desde concepciones liberales, que ven al individuo como el centro de la acción política y dejan de lado la idea de colectividad.

3 En ella confluyeron diferentes sectores y movimientos sociales con el objetivo de replantearse la conformación del Estadonación.

4 Escribo en primera persona porque reconozco que los conocimientos son parciales, situados, específicos y localizables (Haraway, 1991), en consecuencia, parto de mi experiencia frente al tema de investigación.

5 Para la "continuidad" del proyecto político encabezado por Rafael Correa el proceso electoral 2017 fue clave.

6 En México encontramos las propuestas de las zapatistas, en Bolivia, la experiencia de la Confederación Nacional de Mujeres Campesinas Indígenas Originarias de Bolivia -Bartolina Sisa-, y en Ecuador, el proceso de formación política de mujeres kichwas Ecuarunari -Escuela Dolores Cacuango. 
ha situado como agentes políticos con posicionamientos críticos al movimiento feminista y étnico. En este andar han dejado al descubierto las problemáticas específicas que viven como mujeres en sus pueblos, evidenciando las escasas oportunidades con que cuentan para acceder a espacios de toma de decisiones. Así, una de las particularidades de su lucha por tener derechos, y por su derecho a participar en las instancias de toma de decisiones de sus comunidades, pueblos y en las estructuras estatales, es que articulan una posición política que entrelaza las perspectivas étnica, de género y de clase. Por ello, el paradigma de la interseccionalidad ${ }^{7}$ permite comprender las circunstancias concretas y las formas específicas en que se combinan y se construyen mutuamente las diferentes categorías como el género, la raza, la clase y la nación, para producir jerarquías o matrices de dominación (Collins, 1998).

Si bien podemos hablar de avances importantes en materia de reconocimiento y conquistas de las mujeres indígenas, ${ }^{8}$ aún existen numerosos retos para lograr una mayor equidad de género; por lo tanto, considero necesario cuestionar los avances formales y materiales registrados en el país y continuar pensando en las otras formas de participación que ellas protagonizan, ya sea como autoridades, asambleístas, o dirigentes de organizaciones mixtas o de mujeres.

\section{Antecedentes y escenarios de participación}

Ecuador es un país pluricultural; el Consejo de Desarrollo de las Nacionalidades y Pueblos del Ecuador (Codenpe) reconoce 18 pueblos indígenas y 14 nacionalidades, los cuales se estiman en 1018176 personas, que representan $7 \%$ de la población total según datos del Censo de Población y Vivienda 2010 realizado por el Instituto Nacional de Estadística y Censos (INEC, 2010); por su parte, las mujeres indígenas constituyen 50.9\% de la población indígena del país (CEPAL, 2013). A pesar de la diversidad que convive en el territorio ecuatoriano, las desigualdades estructurales y el racismo son problemáticas que afectan sistemáticamente a la nación.

La década de los noventa fue paradigmática para los pueblos indígenas latinoamericanos, ya que a lo largo del continente se gestaron protestas y movilizaciones, por ejemplo en Bolivia (1990) y México (1994). En el caso ecuatoriano, las movilizaciones de mayor trascendencia tuvieron lugar en 1990, 1992, 1994, 1997 y 1998; prácticamente toda la década estuvo marcada por una amplia movilización indígena (Barrera, 2001). Los diversos levantamientos indígenas que se desarrollaron en América Latina formaron parte de la campaña continental "500 años de resistencia indígena”, cuya finalidad era avanzar en derechos civiles, políticos, económicos, sociales y culturales (Cabrero, 2013), y establecer una nueva relación con el Estado (Bengoa, 2000; Valladares y Escobar, 2014).

La aparición del movimiento indígena ecuatoriano como un actor político central ocurrió en esa década; no obstante, el proceso comenzó a gestarse desde los sesenta con articulaciones del movimiento campesino y grupos evangelistas. El momento considerado clave en la historia del movimiento fue la fundación de la Confederación de Nacionalidades Indígenas del Ecuador (Conaie) en 1986, que conjuntó a los indígenas de la sierra -Ecuador Runacunapac Riccharimui (Ecuarunari)-y a los de la Amazonia-Confederación de las Nacionalidades Indígenas de la Amazonia Ecuatoriana (Confeniae) - (Resina, 2012). La revitalización de lo étnico y lo cultural dio paso a un proceso de articulación de diferentes pueblos y nacionalidades indígenas, los cuales demandaban al Estado nacional su reconocimiento a partir de su identificación específica, así como el reconocimiento de sus derechos individuales y colectivos.

El discurso de la diferencia trajo consigo un gran potencial movilizador, pues rompió con la idea "de hombres y mujeres homogéneos, unidimensionales. Las personas no son sólo socialistas o conservadoras, sino también son feministas, ecologistas o pacifistas; tampoco pertenecen a una misma agrupación ni se adhieren estable y de manera fija a los mismos valores" (Arditi, 2000: 104); son sujetos plurales que se constituyen a partir de múltiples identidades y se construyen en torno a ellas. Por un lado, el aporte principal del movimiento indígena ecuatoriano de los noventa fue visibilizar las problemáticas que vivían las poblaciones indígenas, acotadas por el racismo y la discriminación (Cervone, 1999); por otro lado, en el plano formal se logró el reconocimiento del Ecuador como un Estado pluricultural y multiétnico en la

7 Viveros (2016) menciona que Hill Collins es la primera en proponer la interseccionalidad como un paradigma alternativo al antagonismo positivismo/posmodernismo, el cual requiere de una teoría normativa e investigación empírica.

8 Por ejemplo, en la Constitución Política del Ecuador, en el artículo 57, en el cual se reconocen los derechos colectivos para los pueblos indígenas, se especifica que el derecho consuetudinario no podrá vulnerar los derechos constitucionales de las mujeres, y tendrá como finalidad promover la igualdad y equidad entre mujeres y hombres. 
Constitución de 1998. En estas discusiones, los pueblos y nacionalidades indígenas se hicieron presentes, y las mujeres indígenas fueron parte de los levantamientos y las movilizaciones, pero no así de la toma de decisiones, donde unas cuantas pudieron visibilizarse y acceder a cargos de representación. Por ejemplo, de las 243 organizaciones indígenas establecidas en Chimborazo, sólo siete tenían como presidenta a una mujer (Cucuri, 2007).

La agenda del movimiento indígena tuvo como característica principal el posicionamiento de las demandas étnicas. Autores como Ospina (2006) y Safa (2008) señalan que la dimensión de género no fue excluida de la agenda del movimiento indígena, sin embargo, fue marginada. En este escenario, las indígenas decidieron constituir organizaciones exclusivas de mujeres, que centraban sus reflexiones y debates en las dificultades que ellas vivían dentro y fuera de sus comunidades. Esto, por supuesto, sin romper o alejarse de las reivindicaciones del movimiento indígena, ya que las demandas de género también forman parte de la defensa de los derechos étnicos, el territorio, el reconocimiento de saberes ancestrales y tradicionales, al incorporar la perspectiva y las preocupaciones de las indígenas sobre temáticas que las afectan de forma directa (Dixon y Gómez, 2009); sin dejar de lado el papel de reproductoras biológicas, culturales y sociales, inmersas en lógicas de participación con un rol protagónico en sus comunidades.

En los niveles parroquial, cantonal y regional, las organizaciones comenzaron a hacer eco en las decisio-

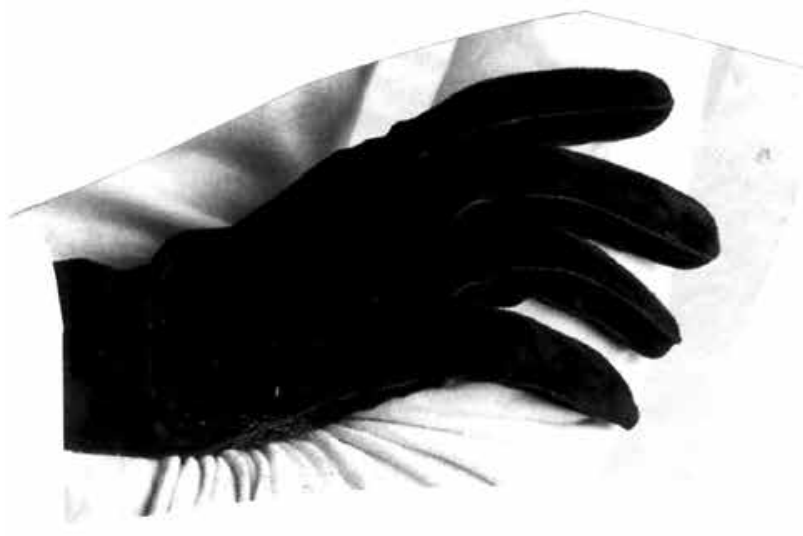

nes que se tomaban en el plano nacional. Las condiciones de exclusión que enfrentaban las indígenas no sólo ocurrían respecto a la sociedad nacional o mestiza, sino que en el interior de sus propias organizaciones y comunidades había diferencias que las colocaban en circunstancias de subordinación. Al respecto, una de las dirigentes de Ecuarunari comentó en una entrevista:

Siempre hemos estado participando en la lucha de las mujeres y participado en las actividades, en las asambleas, en las comunidades, en las mingas, ${ }^{9}$ las mujeres nos organizábamos para una lucha. Por ejemplo, que recibían tanta explotación las mujeres, que nosotras éramos las que levantábamos, así participábamos conjuntamente con compañeros varones y eso nos dio bastante ánimo de decir y seguir organizando y participando [C. Lozano, comunicación personal, 11 de julio de 2013].

La activa intervención de las mujeres indígenas logró que su agenda fuera colocada en la discusión del movimiento, con el fin de incorporarlas a los procesos de toma de decisiones de los que habían sido relegadas y excluidas. Estos espacios tuvieron como principal eje las reflexiones y acciones surgidas desde las propias mujeres; por ello, el ejercicio político de las indígenas nos acerca a la discusión de ciudadanía y derechos, además de una serie de contenidos que vinculan los ámbitos institucionales, sociales y comunitarios en donde se involucran las acciones de los individuos y las colectividades (Pérez y Pinchulef, 2015).

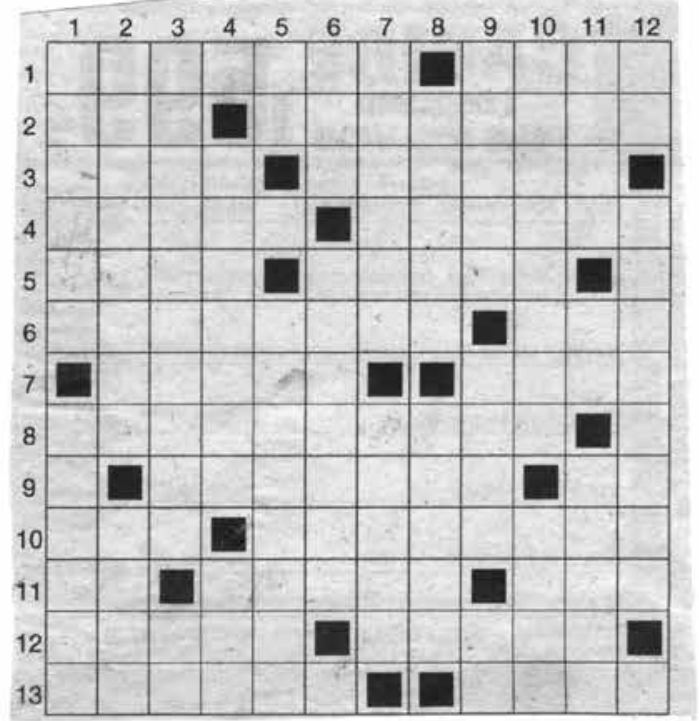

\footnotetext{
9 La minga o minka es una institución de reciprocidad vigente en las sociedades andinas, consiste en el trabajo mancomunado y solidario de los miembros de la comunidad, con la finalidad de contribuir al bien común (De la Torre y Sandoval, 2004).
} 


\section{Configuraciones de la participación}

El movimiento indígena en Ecuador apostó por diversas maneras de encauzar sus demandas; una de ellas fue el apoyo a candidatos indígenas para ocupar cargos en la esfera local (Instraw, 2006). En 1995 se creó el Movimiento de Unidad Plurinacional Pachakutik, el cual se constituyó debido a una reforma electoral que permitía a los movimientos sociales presentar candidatos en las elecciones de 1996 (Cabrero, 2013). Ese mismo año, Auki Tituaña llegó al gobierno local de Cotacachi a través de Pachakutik, y puso en marcha uno de los modelos más exitosos de democracia participativa, la cual significó para algunos autores "una metodología ${ }^{10}$ única a ser replicada en cualquier parte del país o del continente, pues encierra grandes aprendizajes para los pueblos que quieran alcanzar sus propias metas" (Herrera y Machuca, 2015: 15). En este entramado de complejidades, el movimiento indígena se convirtió en un sujeto político legitimado en la construcción de la democracia electoral.

Si bien en el ámbito electoral se dieron algunos pasos importantes, Nina Pacari apunta que los partidos políticos no se han interesado en impulsar la participación de las mujeres indígenas, incluso Pachakutik ha tenido una escasa representación femenina, ya que por lo general los candidatos continúan siendo varones (Instraw, 2006). Por ejemplo, en el periodo 2009-2013, en la Asamblea Nacional del Ecuador dos mujeres indígenas ocuparon la posición de asambleístas; mientras que en el de 2013-2017 fueron cuatro, tres pertenecientes al oficialismo ${ }^{11}$ (Movimiento Alian- za País) y una al Movimiento de Unidad Plurinacional Pachakutik.

Desde la década de los noventa, la inestabilidad y la crisis económica en el país sudamericano tuvieron como consecuencia una serie de derrocamientos y golpes de Estado, en los cuales los movimientos sociales fueron los protagonistas de la resistencia al neoliberalismo. Por ello, tras la coyuntura provocada por la Rebelión de los Forajidos, ${ }^{12}$ el presidente en turno, Lucio Gutiérrez, fue derrocado el 20 de abril de 2005. Luego de estos acontecimientos, en 2006 la figura de Rafael Correa se posicionó con la presentación de su candidatura a la Presidencia de la República mediante el Movimiento Alianza País, el cual fue respaldado por diversos movimientos y partidos políticos de izquierda entre los que se encontraba Pachakutik.

Una de las insignias de la campaña de Correa fue el llamado a una consulta popular para escribir una nueva constitución política. El 15 de enero de 2007 Correa llegó a la Presidencia del Ecuador, momento en que inició la posteriormente denominada Revolución Ciudadana. El 30 de enero de 2007 se instauró en Montecristi la Asamblea Constituyente con 130 asambleístas, quienes a través de mesas de diálogo y discusión se encargaron de redactar el nuevo documento que sustituiría a la Constitución de 1998.

Los pueblos y mujeres indígenas estuvieron presentes en estas discusiones, lo cual se vio reflejado en el reconocimiento de la interculturalidad y la plurinacionalidad -discurso que rompía con la metanarrativa que imaginó la correspondencia entre una nación y un Estado (Pérez, 2015)-, y en la incorporación del

\section{Cuadro 1}

Mujeres indígenas en espacios legislativos

\begin{tabular}{|c|c|c|c|c|c|}
\hline Periodo & Total de legisladores & Cuota de género* & Hombres & Mujeres & Mujeres indígenas \\
\hline 1998-2003 & 121 & $30 \%$ & 105 & 16 & 1 \\
\hline 2003-2007 & 100 & $35 \%$ & 83 & 17 & 1 \\
\hline 2007-2009 & 130 & $45 \%$ & 85 & 45 & 2 \\
\hline 2009-2013 & 124 & $50 \%$ & 84 & 40 & 2 \\
\hline 2013-2017 & 137 & $50 \%$ & 80 & 57 & 4 \\
\hline
\end{tabular}

* Se refiere a la cuota de género vigente para ese periodo.

Fuente: Elaboración propia a partir de información del Consejo Nacional Electoral y la página oficial de la Asamblea Nacional.

${ }^{10}$ Entiéndase en el sentido de la creación de procesos participativos a través de la incorporación de organizaciones, movimientos sociales y la discusión del presupuesto en asambleas (Ortiz, 1999).

${ }^{11}$ Fuerzas políticas que se adhieren o forman parte del grupo político en el poder.

${ }^{12}$ La Rebelión de los Forajidos fue un movimiento golpista protagonizado por las clases medias descontentas con la corrupción de los poderes ejecutivo y legislativo y de los partidos políticos (Acosta, 2005). 
sumak kawsay, que en kichwa significa "buen vivir". ${ }^{13}$ El modelo del buen vivir plantea una relación entre la sociedad, la cultura y la naturaleza, mediada o intervenida a través del Estado, entendido éste como el gobierno y proyecto político vigente.

Respecto a la participación de las mujeres en los sistemas políticos electorales, su incorporación ha ido en ascenso, debido a que desde 1997 se introdujeron en el país los sistemas de cuotas, estableciendo porcentajes mínimos obligatorios, los cuales aumentarían año con año a fin de transitar a la paridad. En 2007, el incremento porcentual se estableció en 50\%; mas fue en la Constitución de 2008 que la paridad se consagró como principio igualitario básico para la confor mación de listas y fórmulas electorales.

Las indígenas no quedaron fuera de las discusiones y buscaron alianzas con otras mujeres, con el propósito de impulsar sus propias agendas, desde una perspectiva de género y étnica. Por ello, las indígenas ecuatorianas participaron con los diferentes movimientos de mujeres del país (Arboleda, 2006), a través de los cuales articularon sus exigencias. Esta alianza se concretó con la Asamblea de Mujeres Populares y Diversas del Ecuador, con quienes estuvieron en las discusiones llevadas a cabo en Montecristi (Pérez, 2015). Algunas compañeras del Comité Central de Mujeres Unorcac (CCMU), organización con presencia en el cantón de Cotacachi, recuerdan con orgullo su aporte en los debates de la Constituyente:

En el caso de Cotacachi y Sucumbios hemos logrado poner el artículo $171,{ }^{14}$ donde dice que la justicia ancestral o comunitaria será con la participación de la mujer Por lo que sí hemos logrado incidir en esos espacios además de seguir trabajando en el tema de la economía solidaria y el código penal [M. Fueres, comunicación personal, 11 de abril de 2014].

Este logro es trascendental; constituyó la primera vez que las indígenas fueron protagonistas en la discusión de leyes que afectan su vida cotidiana; además de señalar que el tema de la justicia es central en la construcción del Estado plurinacional, al reconocer las particularidades de los pueblos indígenas en la impartición de justicia y, como menciona Boaventura de Sousa Santos: “Tomar en serio la justicia indíge- na es tomar en serio el proyecto de transformación pluralista, descolonizadora y democratizadora de la sociedad y del Estado" (2012: 46). En el caso ecuatoriano, donde las dificultades que las mujeres enfrentan dentro de la justicia comunitaria son vastas (Pérez, 2015), este reconocimiento formal resulta significativo y comprometido con las problemáticas de los pueblos y las mujeres. En este sentido y respecto a otros lugares de América Latina, en Ecuador las cosas han cambiado por lo menos en el nivel legislativo, aunque, en la práctica, los resultados siguen siendo diversos.

\section{Llegó la Revolución Ciudadana}

Sin duda, el logro más destacado del movimiento indígena fue la consagración de varios preceptos en la Constitución de 2008, la cual transformó los cimientos del Estado ecuatoriano y dio pie a la legitimación del mandato de Rafael Correa, que se distinguió por el creciente giro a la izquierda protagonizado por diferentes países de la región, así como el inicio del llamado periodo posneoliberal. ${ }^{15}$ "Los regímenes posneoliberales en sus primeros años sostuvieron que la ciudadanía posmulticultural, se caracterizaba por combinar lo ganado en el periodo neoliberal, el reconocimiento y la participación, con un mayor énfasis en la redistribución" (Martínez, 2014: 105). En esta etapa, el objetivo era configurar una nueva relación del ciudadano con el Estado.

La etapa posneoliberal en Ecuador dio lugar a un tipo de ciudadanía particular, cimentada en el retorno a una clase de indigenismo que colocó a los pueblos indígenas como receptores y beneficiarios de las políticas gubernamentales (Martínez, 2014), despojándolos de su capacidad de agencia. Autores como Martí i Puig y Bastidas (2012) plantean un antes y un después en los nuevos gobiernos latinoamericanos, marcados por grandes desigualdades y descontentos, producto de las reformas posneoliberales, que en el caso del gobierno de Correa se capitalizaron incorporando las demandas de los movimientos; y éstos, a su vez, aprovecharon la oportunidad política para encauzar sus exigencias.

Según Laclau (2009: 57), "todas las demandas, a pesar de su carácter diferente, tienden a reagruparse,

\footnotetext{
${ }^{13}$ El buen vivir se establece como forma de desarrollo, planteado desde un contexto social, cultural y ambiental, sin reducirlo a parámetros occidentales de progreso, racionalidad, crecimiento y consumo (Gudynas, $2011 \mathrm{~b}$ ).

${ }^{14}$ Dicho artículo establece: "Las autoridades de las comunidades, pueblos y nacionalidades indígenas ejercerán funciones jurisdiccionales, con base en sus tradiciones ancestrales y su derecho propio, dentro de su ámbito territorial, con garantía de participación y decisión de las mujeres".

${ }^{15}$ Se utiliza el término "posneoliberal” siguiendo los trabajos de Martínez (2014) y Dávalos (2014).
} 
y forman lo que denominaremos una cadena equivalencial", en donde no existe una separación tangencial, sino que todas las demandas se articulan en torno a la consolidación de sujetos populares. El resultado de esta confluencia en un primer momento fue la satisfacción, la confianza y la legitimidad, lo cual redujo la protesta social, al disminuir los márgenes de movilización. Empero, la ciudadanía posmulticultural (Martínez, 2014) representó una agudización de problemas estructurales como el racismo y la discriminación, además de la cooptación de líderes indígenas por parte del partido oficial, Movimiento Alianza País.

En los primeros años del mandato de Correa existía una importante aprobación de las acciones implantadas en el país. El gobierno no leyó las propuestas del movimiento indígena como las plantearon los propios actores, y, de hecho, la interculturalidad y la plurinacionalidad fueron comprendidas como una especie de vitrina en la cual se podía mostrar la diversidad, por lo que algunos indígenas ocuparon cargos políticos dentro del gobierno oficial de modo decorativo, sin cuestionar los problemas estructurales que enfrentaban las sociedades indígenas, no sólo en su interior sino respecto a los otros. A pesar de las múltiples contradicciones, es innegable reconocer los avances normativos y legislativos obtenidos en Ecuador en materia de etnicidad. Sin embargo, el papel del Estado frente a las identidades continúa siendo un espacio en disputa, donde los preceptos de la democracia y el mercado acotan la actuación política de los ciudadanos (Pérez, 2015).

A partir de 2010 han surgido nuevas olas de movilizaciones y exigencias de los pueblos y nacionalidades indígenas, que se oponen a políticas del gobierno, generando nuevas tensiones y propuestas (Martíi Puig y Bastidas, 2012). En este aspecto me parece relevante recuperar un fragmento de la entrevista a una lideresa del movimiento indígena:

Ahora al frente de este problema de la política del Estado ecuatoriano está el presidente, desde la política del presidente dice que somos democráticos, la plurinacionalidad, la multiculturalidad, multiétnico, de muchas culturas y somos diferentes con diferentes identidades, diferentes pueblos y tenemos nuestros propios derechos; pero esos derechos están violados en cada momento, los derechos que hemos ganado en la Constitución, los derechos que hemos ganado en los espacios internacionales, los dere- chos colectivos del Convenio 169 de la ort. Ha sido un proceso de lucha del movimiento indígena, pero aun así la Constitución y todos esos derechos están yendo por debajo de todo el gobierno de Rafael Correa [C. Lozano, comunicación personal, 11 de julio de 2013].

En Ecuador ha existido una permanente tutela del mundo occidental, el cual incrementa su intervención en los pueblos indígenas con la finalidad de acotar su actuación política. Parte de la política asumida por el gobierno correísta consistió en incorporar los principales postulados del movimiento indígena, pero con una interpretación conveniente a las necesidades del mercado, bajo el modelo llamado por Correa como posneoextractivista, el cual amplía el giro extractivista con el argumento de reinvertir las ganancias de la explotación de los recursos energéticos y minerales para beneficios sociales (Tabares, 2016). Esto se ha reflejado de manera inmediata en la intervención con fines de explotación de los recursos en los territorios indígenas, por lo que podríamos afirmar que la plurinacionalidad no ha generado cambios reales, como lo apuntó una de las lideresas:

Para nosotros la plurinacionalidad era que somos muchas culturas, pero en iguales condiciones de vida, en armonía, con mucho respeto, tener el espacio de trabajo, de ejercer nuestros propios derechos, hoy en día por ejemplo si hablamos del tema de justicia indígena, la justicia indígena tiene un límite de aquí para acá, de esto para acá ya no solucionan los pueblos indígenas y eso es quitarnos nuestro derecho. Entonces eso no es para nosotros la plurinacionalidad, no es esta contienda, más bien hemos dado herramientas para que Correa pueda hacer su discurso político, nos ha robado todo nuestro trabajo, nuestra lucha, que ahora puede ver que no va a salir, porque no sale [C. Lozano, comunicación personal, 11 de julio de 2013].

Los pueblos y nacionalidades indígenas del Ecuador se encuentran frente a una práctica ficticia de la plurinacionalidad, un reconocimiento delimitado $\mathrm{y}$ reducido de autonomías jurídicas, controladas por el Estado y utilizadas como parte del discurso modernizador. Es decir, estamos frente a un incumplimiento sistemático de leyes y tratados nacionales e internacionales, ${ }^{16}$ que incluyen una reducción a la actuación política de los pueblos indígenas.

${ }^{16}$ La Secretaría Nacional de Planificación y Desarrollo (Senplades) propuso una transformación de la matriz productiva, basada en la explotación de recursos naturales (2012), yendo en contra de los derechos colectivos y de la naturaleza consagrados en la Constitución de 2008, así como los derechos indígenas reconocidos en el Convenio 169 de la Organización Internacional del Trabajo. 


\section{¿Cómo participan las mujeres indígenas?}

Institucionalmente, las indígenas están distanciadas de procedimientos y prácticas gubernamentales (Davis, 1999), de ahí que resulte muy difícil que cuenten con los mecanismos para acceder al ejercicio pleno de sus derechos, lo cual las coloca en una posición subordinada respecto a sus pares. Con todo, y pese a las distancias geográficas y culturales que enfrentan, muchas de ellas han logrado insertarse en espacios políticos, ya sea en organizaciones mixtas, de mujeres, locales o nacionales, así como dentro de las estructuras de gobierno, desde el oficialismo, o bien en movimientos o partidos políticos que forman parte de la oposición. Por tanto, las posiciones que ocupan se convierten en bisagras que permiten la reivindicación de sus derechos y la visibilización de su lucha.

Por ejemplo, según datos del INEC (2010), en el caso de Chimborazo -provincia con mayor población kichwa- en las elecciones seccionales de 2009, de los 315 cargos de elección popular, las mujeres ocuparon 42 puestos políticos en las juntas parroquiales rurales, 14 concejalías y una viceprefectura, lo cual representa $18 \%$ de los cargos en toda la provincia. Por otra parte, en las elecciones de 2013, las candidaturas indígenas tuvieron una mayoría de mujeres, como lo muestra el cuadro 2 .

Cuadro 2

Candidaturas indígenas 2013

\begin{tabular}{lcc}
\hline Candidaturas & Mujeres & Hombres \\
\hline Provinciales & 21 & 8 \\
$\quad$ Sierra & 2 & 4 \\
$\quad$ Amazonía & 3 & 2 \\
Nacionales & 1 & 1 \\
Parlamento Andino & 27 & 15 \\
\hline Total & & \\
\hline
\end{tabular}

Fuente: Sánchez (2013: 22).

Desde su posición, las indígenas colocan en la agenda los temas de su interés, empero, como señalaría una dirigente kichwa, "cuando las mujeres nos organizamos y participamos, no sólo luchamos por nuestros derechos, sino también por los derechos de toda la comunidad" (L. Andrade, comunicación personal, 24 de abril de 2014). Esto nos habla de agendas que no están desvinculadas, pues, si bien las indígenas posicionan temas que tienen que ver con salud, violencia de género, economía, cuidado del medio ambiente y educación (Pérez, 2015), vinculan cada temática con sus experiencias comunitarias, familiares y personales. Las indígenas ocupan cargos, contienden por candidaturas, forman parte de organizaciones y posicionan sus demandas; sin embargo, persiste el sexismo; muchas no llegan a ser protagonistas y tienen que conformarse con ser suplentes, sustitutas o parte de las dirigencias, pero sin ser las principales voceras.

Hablar de participación política en el caso de las mujeres indígenas nos permite sumergirnos en múltiples escenarios y deconstruir los conceptos clásicos u occidentales de política, los cuales tienden a enfatizar la relación del Estado con las instituciones, dejando de lado las relaciones y prácticas de gobernantes y gobernados. Al recuperar la voz de lideresas indígenas se pueden visualizar las formas en que ellas conciben la política: 1) aquellas que la consideran como un espacio vinculado con la corrupción, 2) las que la perciben como exclusiva de los sistemas políticos-electorales y los espacios institucionales, y 3) aquellas que la asocian con procesos de toma de decisiones que se ejercen en la familia, la comunidad y las organizaciones (Pérez, 2015). Es interesante conocer estas miradas, sobre todo porque incluso las indígenas que ven la política como una actividad desprestigiada se encuentran pugnando por acceder a la toma de decisiones, lo cual nos habla de mujeres ejemplares que muchas veces cuestionan las prácticas tradicionales, y muchas otras, desde los propios discursos, como el de la complementariedad, ${ }^{17}$ posicionan sus demandas.

Para el Ecuador, 2017 fue un año decisivo no sólo para el entonces presidente Rafael Correa, sino también para la denominada Revolución Ciudadana, debido a la existencia de grupos que apoyaban algunas de las iniciativas neoextractivistas emprendidas en los territorios indígenas, frente a grupos comprometidos con la defensa de la vida y el territorio. ${ }^{18}$

${ }^{17} \mathrm{El}$ principio fundamental del orden del cosmos andino es el dualismo asimétrico, al que denominan yanatin. Los dos polos del yanatin son el masculino, llamado phaña, que corresponde al principio patriarcal, y el femenino, nombrado lloq'e, que corresponde al principio matriarcal (Müller y Müller, 1984: 164). Por lo cual, la complementariedad surge de la idea de un dualismo que permite la existencia de un todo; mas, como señala Marisol de la Cadena, si bien hay una división sexual del trabajo complementaria, la subordinación es subyacente (De la Cadena, 1991).

${ }^{18}$ El extractivismo consiste en un modelo de desarrollo basado en la explotación minera y petrolera. Con la llegada de gobiernos de izquierda a países de la región latinoamericana, se conformó el denominado neoextractivismo; bajo este modelo, el Estado es más activo y con reglas claras. En ocasiones, se renegociaron contratos, regalías y tributos, además de potenciar el papel de empresas estatales (Gudynas, $2011 \mathrm{~b}$ ). 

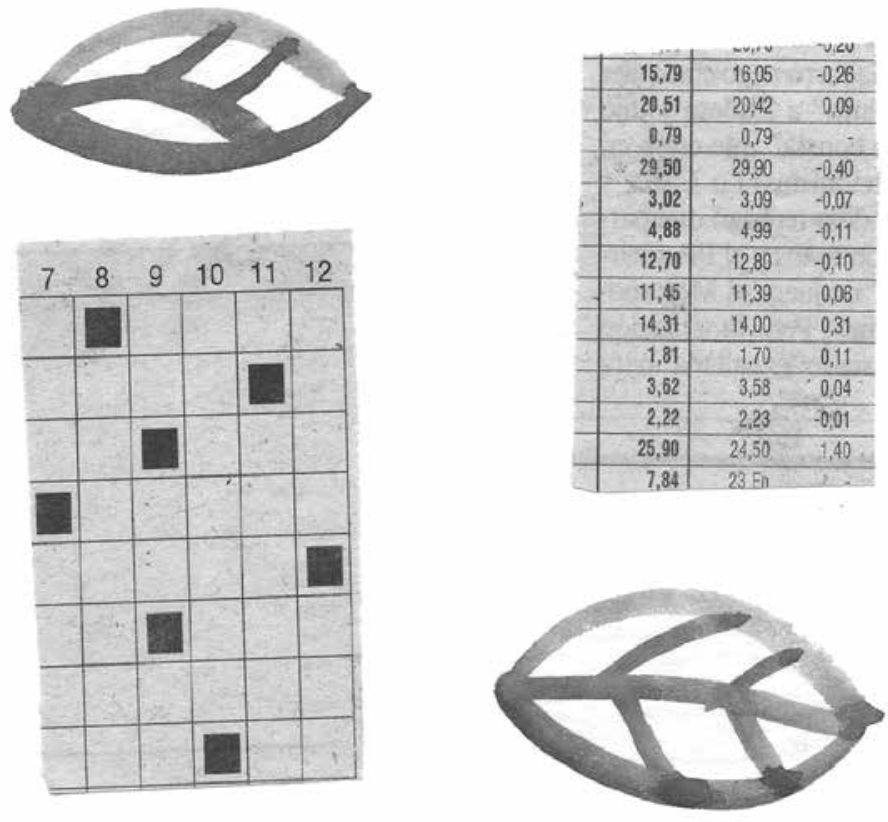

En Ecuador se han impulsado diferentes proyectos de explotación petrolera y minera (extraoficialmente, la organización Minka Urbana indica que, al 2017, $11 \%$ del territorio se ha entregado a mineras transnacionales, lo cual impacta sobre todo a territorios y pueblos indígenas). La Ley de Minería ha sido una de las legislaciones más cuestionadas no sólo por la población indígena, sino también por movimientos ecologistas y la ciudadanía en general. Pese a los cuestionamientos, Rafael Correa señaló: "No daremos marcha atrás en la Ley de Minería, porque el desarrollo responsable de la minería es fundamental para el progreso del país. No podemos sentarnos como mendigos en el saco de oro, afirmó el 15 de enero de 2009" (Gudynas, 2011 a: 87). A partir de 2015, se han gestado nuevas movilizaciones en el país como forma de rechazo a decisiones gubernamentales que van en detrimento de derechos conseguidos por la ciudadanía, las cuales afectan los territorios indígenas. Estas jornadas se han distinguido por una importante participación de mujeres indígenas, quienes han recibido una fuerte ofensiva criminalizante por parte del gobierno.

Pese a los logros en materia de reconocimiento de derechos de los pueblos indígenas, la pregunta es cómo hacer efectivo el ejercicio de derechos. Para algunas lideresas, son varios los elementos que dificultan ejercer sus derechos, entre otros: 1) acceso a educación, 2) carencia de recursos económicos y oportunidades, y 3) ausencia de formación política. No obstante, un número elevado de ellas considera que sus organizaciones de base les han permitido crear pro- puestas de trabajo, adquirir conocimientos, compartir experiencias políticas, religiosas y culturales, así como articularse a redes nacionales e internacionales de movimientos indígenas (Pérez, 2015). De acuerdo con una de las lideresas kichwas, ahora las indígenas tienen mayor capacidad de organización, sin embargo, deben dejar de ser vistas como beneficiarias, pues, al igual que los hombres, cuentan con capacidad de agencia. Para ella es relevante que las políticas públicas se construyan de manera participativa y desde las necesidades territoriales (M. Piñan, comunicación personal, 10 de febrero de 2017).

Una vez que las indígenas se incorporan a organizaciones y participan en la esfera pública, difícilmente abandonan las filas de los espacios donde militan o llevan a cabo sus actividades. Por un lado, muchas de las compañeras han expresado las dificultades que deben superar dentro de sus familias, ya que participar no las excluye del cumplimiento de los roles que su cultura les ha asignado, como el cuidado del hogar y de los niños, lo cual triplica el esfuerzo que deben efectuar. Por otro lado, se enfrentan a mayores obstáculos y requerimientos para que su desempeño político y organizativo sea reconocido; por ejemplo, una dirigente, desde su propia experiencia y trayectoria participativa, comenta:

Cuando una mujer es líder no porque sea líder, no se deja de ser madre, no porque es líder se deja de ser esposa, no porque es líder se deja de trabajar en la agricultura, que hace artesanía, que prepara comida, que hace ropa, todo eso no se deja de hacer, todo es un conjunto de actividades. Creo que una mujer que está atenta a todo esto tiene doble carga, pero esa carga a nivel de los políticos, a nivel de las autoridades no se reconoce, nunca valoran eso [C. Lozano, comunicación personal, $11 \mathrm{de}$ julio de 2013].

Así pues, las condiciones de participación a las que se enfrentan las indígenas son mucho más complejas. No cumplir con sus roles tradicionales o cuestionarlos las coloca en una posición que muchos varones indígenas han llamado separatista o desleal a la causa étnica; pero el problema continúa siendo que no se reconoce ni se valora el esfuerzo que ellas hacen para cumplir con todas sus actividades. Cabe apuntar que esta serie de avances y logros legislativos debe ir acompañada de programas y políticas públicas que ayuden a hacer efectivo el ejercicio de derechos, sin dejar de cuestionar el papel del Estado, que se convierte en punta de lanza en el reconocimiento de identidades, pero al mismo tiempo se sitúa como el principal techo de cristal que reproduce ciudadanías indígenas limitadas. 


\section{Consideraciones finales}

La incorporación de las mujeres y de las mujeres indígenas en la toma de decisiones y en la ocupación de cargos gubernamentales es parte de un proceso gradual acotado por un conjunto de factores ideológicos, culturales e históricos que han repercutido en la invisibilización de las mujeres en la esfera pública, factores que se acentúan de forma exponencial cuando ser indígena está presente. Por tal motivo, es necesario que las indígenas incorporen, asuman y ejerzan los derechos que les corresponden como miembros de un pueblo indígena, como mujeres y en general como sujetos y ciudadanas, haciendo uso de las prerrogativas que les pertenecen por el hecho de ser integrantes de un Estado-nación.

La revitalización de identidades por parte de las mujeres indígenas está incluida en una estrategia que permite discutir el tema de las identidades, las cuales trascienden los marcos analíticos para convertirse en herramientas políticas, con la posibilidad de cuestionar las relaciones de poder existentes en las estructuras sociales. Situarse desde una o múltiples identidades ayuda a dar contenido a las demandas y reivindicaciones que diversos sectores subalternos, como las mujeres y los pueblos indígenas, articulan para expresar su rechazo a las formas que por muchos años han sido el motor desde el cual opera la opresión.

La identidad de las mujeres indígenas en Ecuador no puede ser pensada sin el tema de la identificación, o la asignación de una posición subordinada frente al mundo occidental o frente a sus propias comunidades, por ende, para las indígenas, la reivindicación de lo étnico no se agota en el reconocimiento por parte del Estado nacional, sino que cuestiona los propios entramados sociales gestados en el interior de los pueblos indígenas. Por tal razón, la perspectiva de género y específicamente el paradigma de la interseccionalidad nos ayudan a cuestionar las categorías esencialistas de la identidad, con el objetivo de comprender cómo las formas de poder y opresión se cruzan para normalizar la subordinación.

El Estado ecuatoriano ha sido caracterizado por sus avances normativos y legislativos en materia de pueblos indígenas, sin embargo, es imperioso pensar críticamente el papel que éste debe jugar en la apuesta por la reivindicación de lo étnico, cuestionando los remanentes coloniales y los límites de los marcos jurídicos. Hoy en día, la visión y los planteamientos de los pueblos y nacionalidades indígenas no se encuentran en el proyecto de nación ecuatoriano, situación que no difiere de la de las mujeres indígenas. Si bien en el discurso se ha alcanzado un grado de empatía, el racismo y el sexismo las continúan marginando.

Es importante señalar que la Revolución Ciudadana ha sido paradigmática en cuanto a reconocer un marco jurídico de participación e inclusión de las diversidades. Mas, cuando se trata de ejercer derechos, los ciudadanos no cuentan con elementos de actuación. En este aspecto, existe una clara contrariedad, pues los temas que el propio Estado visibilizó al principio del mandato de Rafael Correa son los mismos que contradijeron su política de desarrollo económico. Resulta problemático que las causas indígenas sean una bandera utilizada como capital político electoral y no como una visión que transforme las maneras de hacer política. En consecuencia, pareciera haber un reconocimiento a medias, de tal suerte que, cuando se emprenden políticas encaminadas al desarrollo del país, los indígenas quedan al margen de las discusiones.

Si bien las mujeres indígenas se integran a las exigencias de sus pueblos, que se han traducido en la búsqueda de derechos y reconocimiento, ellas también se sitúan desde su posición como mujeres, discutiendo los roles que tradicionalmente les han sido asignados y apelando a la importancia que ellas tienen dentro de sus culturas. En este sentido, es fundamental la creación de espacios donde las mujeres expongan y debatan sus condiciones particulares, con la finalidad de introducir mecanismos para que las agendas de las indígenas se vean incorporadas a la lucha del movimiento indígena, además de establecer puentes con otros sectores de la sociedad, que contribuyan a la construcción de los pueblos indígenas como actores políticos centrales en la escena nacional.

\section{Fuentes}

Acosta, Alberto

2005 "Ecuador: ecos de la rebelión de los forajidos", Arboleda, María en Nueva Sociedad, núm. 198, pp. 42-54.

2006 "Género y gobernanza territorial en Cotacachi y Cotopaxi”, en Pablo Ospina (coord.), Las fisuras del poder: movimiento indígena, cambio social y gobiernos locales, Instituto de Estudios Ecuatorianos-Consejo Latinoamericano de Ciencias Sociales, Quito, pp. 151-214.

ARditi, BenJAMín

2000 El reverso de la diferencia. Identidad y política, Nueva Sociedad, Caracas, 224 pp.

\section{Barrera, Augusto}

2001 Acción colectiva y crisis política: el movimiento indígena ecuatoriano en la década de los noventa, Abya-Yala/Observatorio Social de América Latina/Centro de Investigaciones CIUDAD, Quito, 305 pp. 
BengoA, José

2000 La emergencia indígena en América Latina, Fondo de Cultura Económica, México, 346 pp.

Cabrero, Ferrán

2013 "Aproximación teórica. Ejercer derechos, refundar el Estado", en Ferrán Cabrero (coord.), Ciudadanía intercultural. Aportes desde la participación política de los pueblos indígenas en Latinoamérica, Programa de las Naciones

CAdena, Marisol de lA Unidas para el Desarrollo, Quito, pp. 12-103.

1991 “'Las mujeres son más indias': etnicidad y género en el Cusco", en Revista Andina 17, año 9, núm. 1, pp. 7-29.

Cadena, Marisol de la

Y ORIN STARN

2009 "Indigeneidad: problemáticas, experiencias y agendas en el nuevo milenio", en Tabula Rasa, Revista de Humanidades, núm. 10, enero-junio, pp. 191-223.

Canabal, Beatriz

2003 "Mujeres indígenas y democracia. Una primera reflexión desde la montaña de Guerrero", en Revista de Estudios de Género. La Ventana, núm. 18, diciembre, pp. 210-253.

CEPAL

2013 Mujeres indígenas en América Latina: dinámicas demográficas y sociales en el marco de los derechos humanos, Centro Latinoamericano y Caribeño de Demografía-División de Población y División de Asuntos de Género-Comisión Económica para América Latina y el Caribe. Santiago de Chile, 159 pp.

Cervone, Emma

1999 "Introducción", en Emma Cervone y Fredy Rivera Velez (eds.), Ecuador racista: imágenes $e$ identidades, Facultad Latinoamericana de Ciencias Sociales, Sede Ecuador, Quito, pp. 11-18.

Collins, Patricia

1998 "It's All in the Family: Intersections of Gender, Race, and Nation", en Hypatia. A Journal of Feminist Philosophy, vol. 13, núm. 3, agosto, pp. 62-82.

CuCURi, Cristina

2007 Agenda de equidad de género de las mujeres kichwas de Chimborazo, Centro de Desarrollo, Difusión e Investigación Social, Riobamba, $54 \mathrm{pp}$.

Dávalos, PaBlo

2014 Alianza PAIS o la reinvención del poder. Siete ensayos sobre el posneoliberalismo en el Ecua dor, Ediciones Desde Abajo, Bogotá, 422 pp.

Davis, Diane

1999 "The Power of Distance: Re-theorizing Social Movements in Latin America", en Theory and Society, vol. 28, núm. 4, pp. 585-638.

DiXON, BERNARDINE

Y NuRIa Gómez

2009 Estudio de caso Nicaragua. Participación política y liderazgo de las mujeres indígenas en América Latina, Programa de las Naciones Unidas para el Desarrollo, México, 230 pp.

Gudynas, Eduardo

$2011 \mathrm{a}$ "El nuevo extractivismo progresista en América del Sur. Tesis sobre un viejo problema bajo nuevas expresiones", en Alberto Acosta, Eduardo Gudynas, François Houtart, Henry Ramírez
Soler, Joan Martínez Alier y Luis Macas, Colonialismos del siglo XxI. Negocios extractivos $y$ defensa del territorio en América Latina, Icaria Editorial, Barcelona, pp. 75-92.

Gudynas, EDUARDo

$2011 \mathrm{~b}$ "Buen vivir: germinando alternativas al desarrollo", en América Latina en Movimiento, núm. 462, febrero, pp. 1-20.

HARAWAY, DONNA

1991 Ciencia, cyborgs y mujeres. La reinvención de la naturaleza, Cátedra, Madrid, $431 \mathrm{pp}$.

Hernández, Rosalva

2008 "Entre el etnocentrismo feminista y el esencialismo étnico. Las mujeres indígenas y sus demandas de género", en Liliana Suárez y Rosalva Hernández, Descolonizando el feminismo: teorías y prácticas desde los márgenes, Cátedra, Madrid, pp. 170-190.

Herrera, Amparo

y Miguel Machuca

2015 El gobierno local de Cotacachi y los objetivos de desarrollo del milenio relacionados con la salud de la población, Municipio de CotacachiAsamblea de Unidad Cantonal-Consejo Cantonal Intersectorial de Salud, Cotacachi, 37 pp.

INEC

2010 Censo de Población y Vivienda 2010, Instituto Nacional de Estadística y Censos, Quito <http: / /www.inec.gob.ec/estadisticas / > [18 de enero de 2017].

INSTRAW

2006 Participación de las mujeres indígenas en los procesos de gobernabilidad y en los gobiernos locales: Bolivia, Colombia, Ecuador, Guatemala y Perú, Instituto Internacional de Investigaciones y Capacitación de las Naciones Unidas para la Promoción de la Mujer, Santo Domingo, $157 \mathrm{pp}$.

Laclau, ERnesto

2009 "Populismo. ¿Qué nos dice el nombre?", en Francisco Panizza, El populismo como espejo de la democracia, Fondo de Cultura Económica, México, pp. 51-71.

Martí I Puig, SAlvador

Y CRISTINA BASTIDAS

2012 ¿Ha cambiado la protesta? La coyuntura actual de movilizaciones en Bolivia y Ecuador". en Íconos: Revista de Ciencias Sociales, núm. 44 , pp. 19-33.

Martínez, Carmen

2014 "El regreso del indigenismo y el ataque a los derechos indígenas en el Ecuador posneoliberal", en Laura Valladares (coord.), Nuevas violencias en América Latina. Los derechos indígenas ante las políticas neoextractivistas y las políticas de seguridad, Juan Pablos Editor/ Universidad Autónoma Metropolitana-Iztapalapa, México, pp. 105-123.

Müller, Thomas y Helga Müller

1984 "Cosmovisión y celebraciones del mundo andino”, en Allpanchis, vol. XX, núm. 23, pp. 161 172 .

Ortiz, SANTIAGO

1999 "Participación ciudadana y desarrollo local: algunas pistas de reflexión", en Víctor Hugo Torres (ed.), Ciudadanías emergentes: Experiencias democráticas de desarrollo local, AbyaYala, Quito, pp. 63-83. 
OSPINA, PABLO

2006 "Movimiento indígena ecuatoriano, gobierno territorial local y desarrollo económico: los casos del Gobierno Municipal de Cotacachi y el Gobierno Provincial de Cotopaxi”, en Pablo Ospina (coord.), Las fisuras del poder. Movimiento indígena, cambio social y gobiernos locales, Instituto de Estudios EcuatorianosConsejo Latinoamericano de Ciencias Sociales, Quito, pp. 15-118.

Palacios, Paulina

2005 "Construyendo la diferencia en la diferencia: mujeres indígenas en la democracia plurinacional", en Pueblos indígenas, Estado y democracia, Consejo Latinoamericano de Ciencias Pateman, Carole Sociales, Buenos Aires, pp. 311-339.

2009 "Críticas feministas a la dicotomía público/ privado", en Ramiro Ávila et al., El género en el derecho. Ensayos críticos, Ministerio de Justicia y Derechos Humanos / Fondo de Desarrollo de las Naciones Unidas para la Mujer, Quito, pp. 37-65 [1996].

Pegueño, Andrea

2009 "Introducción", en Participación y políticas de mujeres indígenas en América Latina, Facultad Latinoamericana de Ciencias Sociales / Ministerio de Cultura, Quito, pp. 9-25.

Pérez, Lizeth

2015 "Procesos, cambios y rupturas: ciudadanía de mujeres Kichwas en espacios de participación local", tesis de maestría, Facultad Latinoamericana de Ciencias Sociales, sede Ecuador, Quito, 130 pp. <http: / / repositorio.flacsoandes. edu.ec/handle/10469/7715\#.WJjJafl97IU> [ 18 de enero de 2017].

Pérez, Lizeth y Carola Pinchulef

2015 "Enfoques críticos a la participación política de las mujeres indígenas en Chile y Ecuador", en UTCiencia. Ciencia y Tecnología al Servicio del Pueblo, vol. 2, núm. 3, diciembre, pp. 130139.

RESINA, JORGE

2012 La plurinacionalidad en disputa: el pulso entre Correa y la CONAIE, Abya-Yala, Quito, $171 \mathrm{pp}$.

SAFA, Helen

2008 "Igualdad en la diferencia", en Mercedes Prieto (ed.), Mujeres y escenarios ciudadanos, Facultad Latinoamericana de Ciencias Sociales / Ministerio de Cultura, Quito, pp. 57-82.
SÁNCHEZ, JosÉ

2013 Los indígenas y la política. Representación y participación electorales: Ecuador 2013, Universidad Politécnica Salesiana/Abya-Yala, Quito, 139 pp.

Santos, Boaventura de Sousa

2012 "Cuando los excluidos tienen Derecho: jus ticia indígena, plurinacionalidad e interculturalidad", en Boaventura de Sousa Santos y Agustín Grijalva (eds.), Justicia indígena, plurinacionalidad e interculturalidad en Ecuador, Fundación Rosa Luxemburgo/Abya-Yala, Quito, pp. 13-50.

Segato, Rita Laura

2011 "Género y colonialidad en busca de claves de lectura y de un vocabulario estratégico decolonial”, en Karina Bidaseca y Vanesa Vázquez (comps.), Feminismo y poscolonialidad. Descolonizando el feminismo desde y en América Latina, Godot, Buenos Aires, pp. 17-48.

Tabares, Gema

2016 "Estratificación de clases y crisis en el movimiento indígena del Ecuador: élites indígenas. El síndrome del "poncho dorado", tesis de doctorado, Universidad Autónoma Metropolitana-Iztapalapa, México, $421 \mathrm{pp}$.

TORRE, LuZ MARÍA DE LA

y Carlos Sandoval

2004 La reciprocidad en el mundo andino. El caso del pueblo de Otavalo, Abya-Yala, Quito, 121 pp.

VALLADARES, LAURA

Y ANTONIO ESCOBAR

2014 "La etnicidad frente a las nuevas violencias en América Latina", en Laura Valladares (coord.), Nuevas violencias en América Latina. Los derechos indígenas ante las políticas neoextractivistas y las políticas de seguridad, Juan Pablos Editor/Universidad Autónoma Metropolitana, México, pp. 63-104.

Viveros, Mara

2016 "La interseccionalidad: una aproximación situada a la dominación", en Debate Feminista, vol. 52, octubre, pp. 1-17 <http: / /dx.doi.org/ $10.1016 /$ j.df.2016.09.005> [21 de marzo de 2017].

Zegada, María Teresa

2012 Indígenas y mujeres en la democracia electoral: análisis comparado, Tribunal Electoral del Poder Judicial de la Federación, México, 72 pp. 